\title{
An update on radiation therapy for brain metastases
}

\author{
Tai-Chung Lam ${ }^{1}$, Arjun Sahgal ${ }^{2}$, Simon S. Lo ${ }^{3}$, Eric L. Chang ${ }^{4}$ \\ ${ }^{1}$ Department of Clinical Oncology, Li Ka Shing Faculty of Medicine, the University of Hong Kong, Hong Kong SAR, China; ${ }^{2}$ Department of \\ Radiation Oncology, Sunnybrook Health Science Centre, University of Toronto, Toronto, ON, Canada; ${ }^{3}$ Department of Radiation Oncology, \\ University of Washington School of Medicine, Seattle, WA, USA; ${ }^{4}$ Department of Radiation Oncology, Norris Cancer Center and Keck School of \\ Medicine at University of Southern California, Los Angeles, CA, USA \\ Contributions: (I) Conception and design: All authors; (II) Administrative support: TC Lam, EL Chang; (III) Provision of study materials or patients: \\ All authors; (IV) Collection and assembly of data: TC Lam; (V) Data analysis and interpretation: All authors; (VI) Manuscript writing: All authors; (VII) \\ Final approval of manuscript: All authors. \\ Correspondence to: Eric L. Chang. Department of Radiation Oncology, Norris Cancer Center and Keck School of Medicine at University of Southern \\ California, Los Angeles, CA, USA. Email: eric.chang@med.usc.edu.
}

\begin{abstract}
Radiation therapy (RT) is an important treatment modality for brain metastases. Recent clinical trials have established the role of stereotactic radiosurgery (SRS) in the improvement of local control of brain metastases. Prospective trial data confirmed the feasibility and efficacy of using SRS alone to treat 5 or more brain metastatic tumors. Besides tumor control, there is increased emphasis on quality of life and neurocognitive function preservation. The new approach of hippocampi-sparing whole brain RT has been tested in an attempt to minimize the neurocognitive toxicity of whole brain RT. There is now level 1 evidence to support the multidisciplinary approach of surgical resection of brain metastases followed by cavity SRS which has been shown to preserve neurocognitive function without compromising survival. The current review summarizes the recent advances in RT for brain metastases.
\end{abstract}

Keywords: Brain metastases; radiation therapy (RT); stereotactic radiosurgery (SRS); whole brain radiation therapy (WBRT)

Submitted Apr 16, 2017. Accepted for publication May 15, 2017.

doi: $10.21037 /$ cco.2017.06.02

View this article at: http://dx.doi.org/10.21037/cco.2017.06.02

\section{Introduction}

Brain metastases occur frequently in patients with cancer and certain cancers such as lung cancer, breast cancer, melanoma, and renal cell carcinoma carry a higher risk. About $30 \%$ of cancer patients will develop brain metastases and the incidence is rising (1). The judicious selection of treatment for patients with brain metastases is crucial to maximize positive outcomes including improvement of survival and preservation of neurocognitive function and quality of life. In the recent years, significant progress has been made to optimize treatment outcomes for brain metastases. As patients survive longer with the use of very effective cytotoxic chemotherapy and targeted therapy, they are more likely to suffer the toxic effects of their brain metastasis treatments. The most recent trials in brain metastases focus on reduction of toxicities of RT for brain metastases while maintaining the overall survival. The scope of this review is to highlight the recent advances of radiation therapy (RT) management of brain metastases based on prospective data.

\section{Further evidence of using SRS alone for brain metastases}

Multiple phase III randomized controlled trials (RCTs) had already confirmed the important role of stereotactic radiosurgery (SRS) in the management of multiple brain metastases (2-5). In the past 3 years, we have had more high quality evidence supporting the use of SRS alone for brain metastases. In 2015, Sahgal and colleagues published an individual patient data meta-analysis of phase 3 trials of SRS 


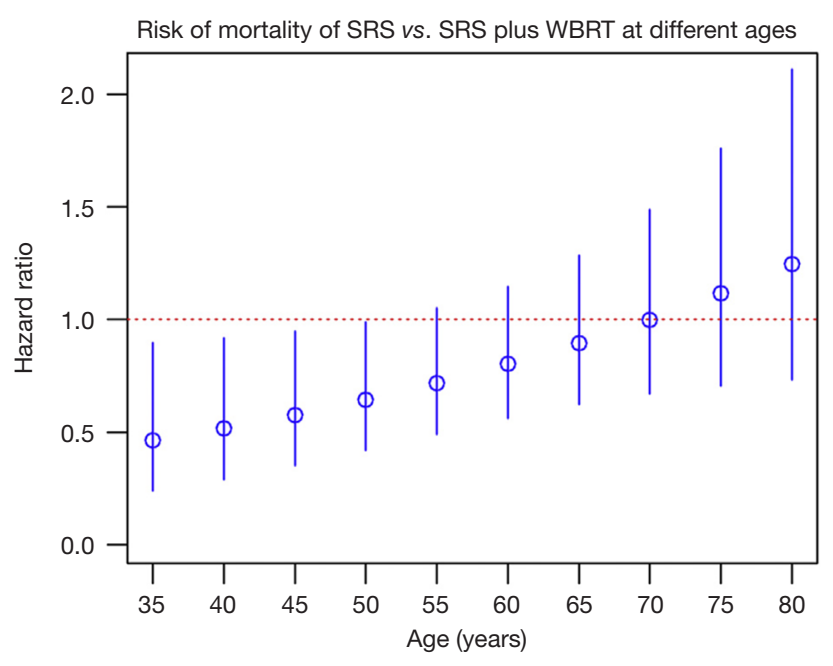

Figure 1 Treatment effects of SRS $v s$. SRS plus WBRT and the corresponding $95 \%$ confidence intervals on survival at 35 to 80 years of age. Figure extracted from Sahgal et al. (6). SRS, stereotactic radiosurgery; WBRT, whole brain radiation therapy.

with or without whole brain radiation therapy (WBRT) for 1-4 brain metastases (6). The study pooled individual data of 364 patients from three RCTs $(2,3,5)$. While it was not surprising that the meta-analysis confirmed that single brain metastasis patients had better overall survival and lower distant brain failure, it was the first report showing that age was an important modifier on treatment outcome. For patients with age $<50$ years old, addition of WBRT to SRS actually resulted in detrimental effect on overall survival. Hazard ratios (HRs) for patients 35, 40, 45, and 50 years of age were $0.46[95 \%$ confidence interval $(\mathrm{CI})=0.24-0.90)$, $0.52(95 \%$ CI $=0.29-0.92), 0.58$ (95\% CI $=0.35-0.95)$, and 0.64 (95\% CI $=0.42-0.99$ ], respectively (Figure 1$)$. In younger patients $<50$ years old, omission of WBRT did not affect distant brain failure rate. The authors hypothesized that those patients were exposed to the adverse effects of WBRT without yielding a therapeutic gain with respect to distant brain relapse rates, which may explain the phenomenon.

In clinical practice, given the observation mentioned above, when we encounter young brain metastases patients who will continue receive active systemic anti-cancer treatments, strong consideration should be given for omission of WBRT.

In 2016, the 4th phase III RCT on SRS with or without WBRT by the Alliance for Clinical Trials in Oncology group (NCCTG N0574) was published (7). Patients with
1-3 brain metastases were randomized to SRS and SRS plus WBRT. The primary endpoint used was cognitive function decline at three months. Cognitive function deteriorated more frequently in patients who received WBRT plus SRS compared with SRS alone, especially in the domains of immediate recall, memory, and verbal fluency. The finding was consistent with previous phase III RCT by M.D. Anderson Cancer Center (3). It has also corroborated the facts that intracranial disease control is improved with the inclusion of adjuvant WBRT (3-month failure rate $24.7 \%$ vs. $6.3 \%$ ) but there is no improvement in overall survival. Given the presence of robust level 1 evidence supporting the omission of WBRT in patients with 1-4 metastases, SRS alone should be the preferred treatment option for patients with limited brain metastases. Compared to WBRT plus SRS, the potential advantages of SRS alone include better preservation of neurocognitive function, better quality of life, rapid delivery as a single outpatient session, minimal recovery time, and minimal delay in the re-initiation of systemic treatments. Furthermore, there is no negative impact on overall survival. In 2014, the American Society for Radiation Oncology (ASTRO) Choosing Wisely ${ }^{\circledR}$ Campaign has issued a statement recommending against routinely adding adjuvant WBRT to SRS for limited brain metastases.

Technological advances in treatment delivery and planning also allows SRS to be given efficiently and safely to patients with more than 4 brain metastases. In 2014, a phase II clinical trial JLGK0901 (8) was published by Yamamoto and colleagues. It was an adequately-powered, prospective, non-inferiority observational cohort study which had recruited patients with newly diagnosed 1-10 brain metastases. The treatment given was SRS alone without WBRT. The limit for total intracranial disease burden was a total volume $\leq 15 \mathrm{~mL}$ and the largest brain metastasis had to be $\leq 10 \mathrm{~mL}$ or $\leq 3 \mathrm{~cm}$ in longest diameter. Among the enrolled patients, the mean of the total volume of brain metastases was approximately $3 \mathrm{~mL}$. More than $85 \%$ of patients in this cohort had Karnofsky Performance Status (KPS) of 80 or above. RPA class 1 patients constituted about $28 \%$ and class 2 patients about $70 \%$.

The result of JLGK0901 showed that the overall survival of patients with 5-10 brain metastases was not inferior to that of 2-4 brain metastases, and the side effect profile was similar between the two groups. The results of this trial corroborated those of other studies that the total volume, but not an arbitrary number of brain metastases, was a more important predictive factor for prognosis $(9,10)$. The main 
limitation was that the study did not have follow-up data on MRI, MMSE, neurocognitive function or quality of life measures. Nevertheless the study challenged the traditional dogma $(11,12)$ that SRS should only be offered to patients with no more than 3-4 brain metastases. It also guided the future direction of clinical trials to compare WBRT and SRS in 4 or more brain metastases. A phase III RCT comparing WBRT vs. SRS alone in 5-15 brain metastases will be performed in North America to confirm the findings of JLGK0901 (ClinicalTrials.gov NCT03075072).

\section{Neurocognitive function preservation}

In the past, the potential impact of RT on cognitive function was largely ignored due to the very poor overall survival of brain metastases patients $(13,14)$. With the advancement of systemic targeted therapy, the outcomes of patients have significantly improved, particularly amongst those with good prognostic factors (15). The routine use of WBRT in patients has drawn much controversy due to its impact on neurocognitive function (16) and quality of life (17).

The most studied approach for neurocognitive function preservation is the omission of WBRT. The Alliance study (NCCTG N0574) (7) has provided important information on the delicate balance of neurocognitive function and intracranial disease control. In the study, the primary endpoint of the study was set low at one standard deviation (SD) change in neurocognitive function tests. So even for SRS alone arm, the neurocognitive deterioration was high at around $64 \%$ vs. $92 \%$ of WBRT plus SRS arm. If the threshold was set at 2-SD change, the rates would drop to $1.6 \%$ vs. $10.6 \%$ (18).

If we consider the 2-SD change in neurocognitive function as clinically significant, is it justified to improve intracranial control by upfront WBRT while taking around $10 \%$ risk of neurocognitive function deterioration? It is important to consider treatment which maximizes intracranial control especially if the local health system cannot arrange for frequent surveillance MRI and prompt salvage treatment for relapse. From the MD Anderson RCT (3), the one-year CNS progression rate was $73 \%$ for SRS alone arm and 27\% for SRS plus WBRT arm $(\mathrm{P}=0.0003)$. For the Alliance study (7), the six-month CNS progression rate of the two arms were $35 \%$ vs. $12 \%$ $(\mathrm{P}<0.001)$.

The importance of maximizing intracranial control was illustrated by the earlier RCT reported by Aoyama et al. $(2,19)$. In this RCT, SRS alone arm actually had worse functional outcome compared with SRS plus WBRT ( $41 \%$ vs. $24 \%$ at 12 months), as measured by a relatively crude scale of mini-mental state examination (MMSE). It was postulated that this was related to the inferior overall intracranial control caused by distant CNS recurrence.

On the other hand, there is active research effort to reduce the neurocognitive impact of WBRT while keeping the higher intracranial control rate. One approach is to use pharmacological agents for neuroprotection. In 2013, Brown et al. reported the results of RTOG 0614 (20), which was a multi-center RCT testing the use of memantine, an $\mathrm{N}$-methyl-D-aspartate (NMDA) receptor antagonist, for protection of neurocognitive function in patients treated with conventional WBRT.

The primary end-point of the study, decline in delayed recall in 24 weeks, was negative. This was possibly due to the low rate of analyzable patients at 24 weeks (149 out of 508 patients) which decreased the power of the study. Among the secondary endpoints, the patients in the memantine arm had significantly longer time to cognitive decline (HR 0.78, $\mathrm{P}=0.01$ ). The patients in the memantine arm also had higher executive function, processing speed and delayed recognition. Overall, memantine was very well tolerated with minimal toxicity. So adding memantine is one of the most practical solutions for neurocognitive function preservation if WBRT is considered necessary.

Another approach to preserve neurocognitive function is by dosimetric sparing of hippocampus with advanced RT planning technique. Hippocampus is a vital structure for memory and learning. It is particularly sensitive to ionizing radiation injury which suppresses neurogenesis of hippocampal neural stem cell (21) and causes neurocognitive deterioration after WBRT. Hippocampal-avoidance whole brain radiation therapy (HA-WBRT) was hence proposed to preserve neurocognitive function in patients with brain metastases.

So far, there was no randomized data supporting the use of HA-WBRT. The most important evidence was from RTOG-0933 (22), which was a phase 2 single arm study published in 2014 comparing the neurocognitive outcomes of patients undergoing HA-WBRT to those of a historical cohort. The primary endpoint was Hopkins Verbal Learning Test-Revised Delayed Recall (HVLT-R DR) at 4 months. The technical requirement of HA-WBRT was carefully defined (23). Planning MRI brain with $\leq 1.5 \mathrm{~mm}$ slice thickness and fusion to planning CT with $\leq 2.5 \mathrm{~mm}$ slice thickness was required. Hippocampus contouring protocol was set for clinicians. Dose to hippocampi was set 
at D100\% $\leq 9$ Gy in 10 fractions and maximum dose $\leq 16$ Gy in 10 fractions. Central rapid plan review was conducted in real time to ensure the plan quality.

Among 113 patients received HA-WBRT, 42 patients were analyzable at 4 months. Mean relative decline in HVLT-R DR from baseline to 4 months was $7.0 \%$ (22) (95\% CI, 4.7-18.7\%), significantly lower in comparison with the historical control of $\sim 30 \%$ (24) $(\mathrm{P} \leq 0.001)$. No decline in QOL scores was observed. The results compared favorably against similar patient group in the M.D. Anderson group RCT (3). Another prospective study at Taiwan reproduced the finding of RTOG 0933 . Forty patients were recruited and 24 of them were available for post-treatment assessment. Dosimetry parameters of hippocampus were correlated to the verbal memory scores of neurocognitive function (25).

The early encouraging results of the HA-WBRT demonstrated that it was technically feasible in clinical practice. However, further implementation in daily practice necessitates evidence from prospective randomized studies. Cost-effectiveness analysis is certainly important and will probably be determining if this technically demanding treatment will be adopted in the era of value-based medicine. Further studies on reproducible and less complex planning technique with lateral opposing beams and central leaf shielding (26) will help resources tight communities to practise HA-WBRT. Currently the NRG group (National Surgical Adjuvant Breast and Bowel Project, Radiation Therapy Oncology Group, and Gynecologic Oncology Group) is conducting the phase 3 CC-001 trial which is recruiting participants to compare Memantine and WBRT with or without hippocampal avoidance in reducing neurocognitive decline in patients with brain metastases (clinicaltrials.gov NCT02360215).

\section{Multidisciplinary approach for brain metastases}

The conventional treatment after surgical removal of brain metastasis was WBRT. Although WBRT after surgical resection has been proven to decrease local failure rate, WBRT failed to improved overall survival $(5,27)$. Due to the lack of overall survival benefit and the concern of neurocognitive function deterioration after WBRT, there has been a trend to treat the surgical cavity with SRS. But in retrospective studies the local control rate of surgical cavity was highly variable from $30-100 \%(28,29)$.

In 2016, three prospective phase 3 randomized studies on surgical cavity SRS were reported in abstract form. The N107C/CEC.3 (30) was a multicenter RCT which recruited 194 patients with 1-4 brain metastases. Patients were randomized to SRS to both the cavity and unresected metastases or WBRT plus SRS to unresected metastases after resection of one lesion. The M.D. Anderson Cancer Center trial by Mahajan et al. (31) included 131 patients with $1-3$ brain metastases and had at least 1 brain metastases completely removed. Subjects were then randomized to SRS to the surgical cavity (or cavities) vs. observation. JCOG0504 (32) was a multi-institutional study from Japan. It recruited 271 patients with $\leq 4$ brain met and one lesion $>3 \mathrm{~cm}$ having been surgically resected. Patients were randomized to observation or salvage SRS for residual and recurrent tumors. The interventions and main results of the three RCTs were summarized in Table 1.

In summary, the three RCTs (30-32) confirmed the safety and efficacy of SRS to surgical cavity. The results were also consistent with the non-surgical RCTs that quality of life and cognitive functions were better preserved in SRS arm (30). However, caution has to be exercised for large lesion with pre-operative diameter $>3 \mathrm{~cm}$ and superficial lesion with meningeal/pial involvement due to higher risk of local failure $(31,33)$.

\section{New evidence on the use of WBRT alone}

WBRT has long been used for palliative treatment since it was first described in 1950s (34). Efficacy of WBRT in palliation of neurological signs and symptoms was reported in early clinical trials in 1980 s $(13,14)$. In resources limited health care systems, it is still the mainstay of treatment of brain metastases (35). However, despite its wide-spread use, the efficacy of WBRT alone for brain metastases in the modern era of effective systemic treatment was still uncertain until the latest publication of QUARTZ study (36) results.

The QUARTZ (quality of life after treatment for brain metastases) study was a non-inferiority, phase 3 study performed in UK and Australian centers. Non-small cell lung cancer patients $(\mathrm{n}=538)$ who were unsuitable for operative intervention or SRS were randomized to the optimal supportive care (OSC) arm with dexamethasone or the WBRT arm. The overall survival, overall quality of life and use of dexamethasone were not significantly different between two arms. The median overall survival times of both arms were around 9 weeks. The quality adjusted life year (QALY) was 46.4 and 41.7 days in WBRT arm and 
Table 1 Randomized controlled trials of post-operative SRS to surgical cavity

\begin{tabular}{|c|c|c|}
\hline Trials & Interventions & Main results \\
\hline N107C/CEC.3 (30) & $\begin{array}{l}\text { SRS to surgical cavity and unresected met } \\
\text { Post-op WBRT and SRS to unresected met }\end{array}$ & $\begin{array}{l}\text { No difference in OS. Long term surgical cavity control better in WBRT } \\
\text { arm. At } 6 \text { months, cognitive decline and QoL were worse in WBRT arm }\end{array}$ \\
\hline Mahajan et al. (31) & $\begin{array}{l}\text { SRS to surgical cavity and unresected met } \\
\text { Observation to surgical cavity and SRS to } \\
\text { unresected met }\end{array}$ & $\begin{array}{l}\text { SRS improved surgical cavity local control ( } 83 \% \text { vs. } 57 \% \text { at } 6 \text { months, } \\
\text { HR 0.4). Pre-op tumor }>3 \mathrm{~cm} \text { associated with worse local control (HR } \\
2.4 \text { ) }\end{array}$ \\
\hline JCOG-0504 (32) & $\begin{array}{l}\text { Salvage SRS to residual or recurrent disease } \\
\text { at surgical cavity } \\
\text { Post-op WBRT }\end{array}$ & $\begin{array}{l}\text { OS similar. Intracranial progression-free-survival longer in WBRT arm } \\
\text { (10.4 vs. } 4.0 \text { months). Non-worsening MMSE and performance scale } \\
\text { similar in both arms at } 12 \text { months }\end{array}$ \\
\hline
\end{tabular}

QoL, quality of life; MMSE, mini-mental state examination; OS, overall survival; SRS, stereotactic radiosurgery; WBRT, whole brain radiation therapy.

OSC arm, respectively. Serious adverse event rates were similar in both arms, but patients in the WBRT arm had more drowsiness, hair loss, nausea, and dry or itchy scalp.

This study suggested that on average WBRT did not improve overall survival, quality of life or dexamethasone use in poor prognostic group lung cancer patients with brain metastases. Yet, there may be subgroups in this heterogeneous population that WBRT may offer advantage in overall survival. Younger patients, particularly those younger than 60 years old, had improved survival with WBRT. There was also non-significant association of overall survival with satisfactory KPS, controlled primary tumor status, good prognostic grouping by recursive partitioning analysis (RPA) (37) and graded prognostic assessment (GPA) (15).

\section{The role of $R T$ in epidermal growth factor receptor (EGFR) mutated lung cancer patients with brain metastases}

The application of QUARTZ data in Asia population is much affected by the high prevalence of EGFR activating mutation in the lung cancer patient populations (38). The survival pattern of EGFR mutated lung cancer patients with brain metastasis is so different that the Diagnosis-Specific Graded Prognostic Assessment (DS-GPA) was updated based on molecular markers (39). The updated DS-GPA, now known as the Lung-molGPA scores, included EGFR and ALK alterations in adenocarcinoma as predictors. The highest GPA score group of 3.5 to 4.0 had a median survival of nearly 4 years.

There was an argument in favor of treating brain metastases with EGFR tyrosine kinase inhibitor (TKI) alone without RT. Despite the known poor drug penetration of blood brain barrier, multiple cohort studies had reported encouraging response rate of brain metastases in the range of 50-90\% with first-generation EGFR TKI alone (40-43).

Meta-analyses were published to address this important clinical problem. The most recent updated one (44) had included 15 studies with more than 1,500 subjects. The results suggested that radiotherapy plus EGFR TKIs gave superior response rate (risk ratio $=1.48$; 95\% CI: $1.12-1.96$, $\mathrm{P}=0.005$ ), prolonged the time-to-CNS-progression (HR $=0.56 ; 95 \%$ CI: $0.33-0.80 ; \mathrm{P}<0.001)$ and overall survival $(\mathrm{HR}$ $=0.58 ; 95 \%$ CI: $0.42-0.74 ; \mathrm{P}<0.001)$ in NSCLC patients with BM. Combined groups, however, had higher rate of incidence of overall adverse effects, especially rash and dry skin.

Magnuson et al. has published a multi-institutional retrospective analysis of TKI naïve EGFR mutated lung cancer patients with brain metastases in 2017 (45). In this cohort of 351 patients, 100 received upfront SRS, 120 received WBRT and 131 received EGFR-TKI alone. Multivariate analysis showed that upfront SRS vs. EGFRTKI, upfront WBRT vs. EGFR-TKI alone, younger age, better performance status, EGFR exon 19 mutation, and absence of extracranial metastases were associated with improved overall survival.

Current evidence, though mainly consists of retrospective studies, supports the early use of RT together with EGFR TKI to achieve maximal intracranial disease control. Evidence from randomized studies is eagerly awaited for this particular condition prevalent in Asian countries. Multiple RCTs are in progress in China (ClinicalTrials. gov NCT01724801, NCY02714010, NCT01887795) and hopefully definitive results will be available soon. 


\section{Summary}

The encouraging results of clinical trials had established the important role of RT in the management of brain metastases. The aim of treatment has evolved from maximizing tumor control to neurocognitive function preservation. In future, multidisciplinary approach to brain metastases with surgery, RT and systemic therapies will further improve the outcome of patients.

\section{Acknowledgements}

None.

\section{Footnote}

Conflicts of Interest: TC Lam and SS Lo have no conflicts of interest to declare. A Sahgal: Past educational seminars with Medtronic, Elekta AB, Accuray Inc., and Varian medical systems; research grant with Elekta $A B$; travel accommodations/expenses by Medtronic, Elekta and Varian; Dr. Sahgal also belongs to the Elekta MR Linac Research Consortium. EL Chang: Speaker honorarium from BrainLab.

\section{References}

1. Frisk G, Svensson T, Backlund LM, et al. Incidence and time trends of brain metastases admissions among breast cancer patients in Sweden. Br J Cancer 2012;106:1850-3.

2. Aoyama H, Shirato H, Tago M, et al. Stereotactic radiosurgery plus whole-brain radiation therapy vs stereotactic radiosurgery alone for treatment of brain metastases: a randomized controlled trial. JAMA 2006;295:2483-91.

3. Chang EL, Wefel JS, Hess KR, et al. Neurocognition in patients with brain metastases treated with radiosurgery or radiosurgery plus whole-brain irradiation: a randomised controlled trial. Lancet Oncol 2009;10:1037-44.

4. Andrews DW, Scott CB, Sperduto PW, et al. Whole brain radiation therapy with or without stereotactic radiosurgery boost for patients with one to three brain metastases: phase III results of the RTOG 9508 randomised trial. Lancet 2004;363:1665-72.

5. Kocher M, Soffietti R, Abacioglu U, et al. Adjuvant wholebrain radiotherapy versus observation after radiosurgery or surgical resection of one to three cerebral metastases: results of the EORTC 22952-26001 study. J Clin Oncol
2011;29:134-41.

6. Sahgal A, Aoyama H, Kocher M, et al. Phase 3 trials of stereotactic radiosurgery with or without whole-brain radiation therapy for 1 to 4 brain metastases: individual patient data meta-analysis. Int J Radiat Oncol Biol Phys 2015;91:710-7.

7. Brown PD, Jaeckle K, Ballman KV, et al. Effect of Radiosurgery Alone vs Radiosurgery With Whole Brain Radiation Therapy on Cognitive Function in Patients With 1 to 3 Brain Metastases: A Randomized Clinical Trial. JAMA 2016;316:401-9.

8. Yamamoto M, Serizawa T, Shuto T, et al. Stereotactic radiosurgery for patients with multiple brain metastases (JLGK0901): a multi-institutional prospective observational study. Lancet Oncol 2014;15:387-95.

9. Yamamoto M, Kawabe T, Sato Y, et al. A case-matched study of stereotactic radiosurgery for patients with multiple brain metastases: comparing treatment results for $1-4$ vs $>/=5$ tumors: clinical article. J Neurosurg 2013;118:1258-68.

10. Baschnagel AM, Meyer KD, Chen PY, et al. Tumor volume as a predictor of survival and local control in patients with brain metastases treated with gamma knife surgery. J Neurosurg 2013;119:1139-44.

11. Soffietti R, Cornu P, Delattre JY, et al. EFNS Guidelines on diagnosis and treatment of brain metastases: report of an EFNS Task Force. Eur J Neurol 2006;13:674-81.

12. Mehta MP, Tsao MN, Whelan TJ, et al. The American Society for Therapeutic Radiology and Oncology (ASTRO) evidence-based review of the role of radiosurgery for brain metastases. Int J Radiat Oncol Biol Phys 2005;63:37-46.

13. Kurtz JM, Gelber R, Brady LW, et al. The palliation of brain metastases in a favorable patient population: a randomized clinical trial by the Radiation Therapy Oncology Group. Int J Radiat Oncol Biol Phys 1981;7:891-5.

14. Borgelt B, Gelber R, Kramer S, et al. The palliation of brain metastases: final results of the first two studies by the Radiation Therapy Oncology Group. Int J Radiat Oncol Biol Phys 1980;6:1-9.

15. Sperduto PW, Kased N, Roberge D, et al. Summary report on the graded prognostic assessment: an accurate and facile diagnosis-specific tool to estimate survival for patients with brain metastases. J Clin Oncol 2012;30:419-25.

16. Meyers CA, Smith JA, Bezjak A, et al. Neurocognitive function and progression in patients with brain metastases treated with whole-brain radiation and motexafin 
gadolinium: results of a randomized phase III trial. J Clin Oncol 2004;22:157-65.

17. Soffietti R, Kocher M, Abacioglu UM, et al. A European Organisation for Research and Treatment of Cancer phase III trial of adjuvant whole-brain radiotherapy versus observation in patients with one to three brain metastases from solid tumors after surgical resection or radiosurgery: quality-of-life results. J Clin Oncol 2013;31:65-72.

18. Mehta MP, Aoyama H, Gondi V. The Changing Role of Whole-Brain Radiotherapy: Demise or Time for Selective Usage? JAMA Oncol 2017;3:1021-1022.

19. Aoyama H, Tago M, Kato N, et al. Neurocognitive function of patients with brain metastasis who received either whole brain radiotherapy plus stereotactic radiosurgery or radiosurgery alone. Int J Radiat Oncol Biol Phys 2007;68:1388-95.

20. Brown PD, Pugh S, Laack NN, et al. Memantine for the prevention of cognitive dysfunction in patients receiving whole-brain radiotherapy: a randomized, double-blind, placebo-controlled trial. Neuro Oncol 2013;15:1429-37.

21. Monje ML, Vogel H, Masek M, et al. Impaired human hippocampal neurogenesis after treatment for central nervous system malignancies. Ann Neurol 2007;62:515-20.

22. Gondi V, Pugh SL, Tome WA, et al. Preservation of memory with conformal avoidance of the hippocampal neural stem-cell compartment during whole-brain radiotherapy for brain metastases (RTOG 0933): a phase II multi-institutional trial. J Clin Oncol 2014;32:3810-6.

23. Kazda T, Jancalek R, Pospisil P, et al. Why and how to spare the hippocampus during brain radiotherapy: the developing role of hippocampal avoidance in cranial radiotherapy. Radiat Oncol 2014;9:139.

24. Mehta MP, Rodrigus P, Terhaard CH, et al. Survival and neurologic outcomes in a randomized trial of motexafin gadolinium and whole-brain radiation therapy in brain metastases. J Clin Oncol 2003;21:2529-36.

25. Tsai PF, Yang CC, Chuang CC, et al. Hippocampal dosimetry correlates with the change in neurocognitive function after hippocampal sparing during whole brain radiotherapy: a prospective study. Radiat Oncol 2015;10:253.

26. van Kesteren Z, Belderbos J, van Herk M, et al. A practical technique to avoid the hippocampus in prophylactic cranial irradiation for lung cancer. Radiother Oncol 2012;102:225-7.

27. Patchell RA, Tibbs PA, Regine WF, et al. Postoperative radiotherapy in the treatment of single metastases to the brain: a randomized trial. JAMA 1998;280:1485-9.
28. Narayana A, Chan K, Brennan C, et al. A phase II trial of stereotactic radiosurgery boost following surgical resection for solitary brain metastases. Journal of Clinical Oncology 2006;24:1552-.

29. Hwang SW, Abozed MM, Hale A, et al. Adjuvant Gamma Knife radiosurgery following surgical resection of brain metastases: a 9-year retrospective cohort study. J Neurooncol 2010;98:77-82.

30. Brown PD, Ballman KV, Cerhan J, et al. N107C/CEC.3: A Phase III Trial of Post-Operative Stereotactic Radiosurgery (SRS) Compared with Whole Brain Radiotherapy (WBRT) for Resected Metastatic Brain Disease. Int J Radiat Oncol Biol Phys 2016;96:937.

31. Mahajan A, Ahmed S, Li J, et al. Postoperative Stereotactic Radiosurgery Versus Observation for Completely Resected Brain Metastases: Results of a Prospective Randomized Study. Int J Radiat Oncol Biol Phys 2016;96:S2.

32. Kayama T, Sato S, Sakurada K, et al. JCOG0504: A phase III randomized trial of surgery with whole brain radiation therapy versus surgery with salvage stereotactic radiosurgery in patients with 1 to 4 brain metastases. J Clin Oncol 2016;34:abstract 2003.

33. Brennan C, Yang TJ, Hilden P, et al. A phase 2 trial of stereotactic radiosurgery boost after surgical resection for brain metastases. Int J Radiat Oncol Biol Phys 2014;88:130-6.

34. Chao JH, Phillips R, Nickson JJ. Roentgen-ray therapy of cerebral metastases. Cancer 1954;7:682-9.

35. Sharma V, Gaye PM, Wahab SA, et al. Patterns of practice of palliative radiotherapy in Africa, Part 1: Bone and brain metastases. Int J Radiat Oncol Biol Phys 2008;70:1195-201.

36. Mulvenna P, Nankivell M, Barton R, et al. Dexamethasone and supportive care with or without whole brain radiotherapy in treating patients with nonsmall cell lung cancer with brain metastases unsuitable for resection or stereotactic radiotherapy (QUARTZ): results from a phase 3 , non-inferiority, randomised trial. Lancet 2016;388:2004-14.

37. Gaspar L, Scott C, Rotman M, et al. Recursive partitioning analysis (RPA) of prognostic factors in three Radiation Therapy Oncology Group (RTOG) brain metastases trials. Int J Radiat Oncol Biol Phys 1997;37:745-51.

38. Midha A, Dearden S, McCormack R. EGFR mutation incidence in non-small-cell lung cancer of adenocarcinoma histology: a systematic review and global map by ethnicity (mutMapII). Am J Cancer Res 2015;5:2892-911.

39. Sperduto PW, Yang TJ, Beal K, et al. Estimating Survival in Patients With Lung Cancer and Brain Metastases: An 


\section{Page 8 of 8}

Update of the Graded Prognostic Assessment for Lung Cancer Using Molecular Markers (Lung-molGPA). JAMA Oncol 2017;3:827-831.

40. Wu YL, Zhou C, Cheng Y, et al. Erlotinib as second-line treatment in patients with advanced non-small-cell lung cancer and asymptomatic brain metastases: a phase II study (CTONG-0803). Ann Oncol 2013;24:993-9.

41. Iuchi T, Shingyoji M, Sakaida T, et al. Phase II trial of gefitinib alone without radiation therapy for Japanese patients with brain metastases from EGFR-mutant lung adenocarcinoma. Lung Cancer 2013;82:282-7.

42. Park SJ, Kim HT, Lee DH, et al. Efficacy of epidermal growth factor receptor tyrosine kinase inhibitors for brain metastasis in non-small cell lung cancer patients harboring either exon 19 or 21 mutation. Lung Cancer

\section{Lam et al. Update on radiation therapy for brain metastases}

2012;77:556-60.

43. Gerber NK, Yamada Y, Rimner A, et al. Erlotinib Versus Radiation Therapy for Brain Metastases in Patients With EGFR-Mutant Lung Adenocarcinoma. Int J Radiat Oncol Biol Phys 2014;89:322-9.

44. Jiang T, Min W, Li Y, et al. Radiotherapy plus EGFR TKIs in non-small cell lung cancer patients with brain metastases: an update meta-analysis. Cancer Medicine 2016;5:1055-65.

45. Magnuson WJ, Lester-Coll NH, Wu AJ, et al. Management of Brain Metastases in Tyrosine Kinase Inhibitor-Naïve Epidermal Growth Factor ReceptorMutant Non-Small-Cell Lung Cancer: A Retrospective Multi-Institutional Analysis. J Clin Oncol 2017;35:1070-7.
Cite this article as: Lam TC, Sahgal A, Lo SS, Chang EL. An update on radiation therapy for brain metastases. Chin Clin Oncol 2017;6(4):35. doi: 10.21037/cco.2017.06.02 